\title{
UNCERTAINTY ANALYSIS OF SUB-EXERCISES IN UAM-SFR BENCHMARK WITH THE MCS CODE
}

\author{
Yunki Jo ${ }^{1}$, Vutheam Dos ${ }^{1}$, Nhan Nguyen Trong Mai ${ }^{1}$, Hyunsuk Lee ${ }^{1}$, and Deokjung Lee ${ }^{1 *}$ \\ ${ }^{1}$ Department of Nuclear Engineering, Ulsan National Institute of Science and Technology \\ 50 UNIST-gil, Ulsan, 44919, Republic of Korea
}

yunki.jo@unist.ac.kr, dosvutheam8@unist.ac.kr,mainhan@unist.ac.kr, hyunsuklee@unist.ac.kr, deokjung@unist.ac.kr*

\begin{abstract}
Uncertainty analysis in Modelling (UAM) for Design, Operation and Safety Analysis of Sodium-cooled Fast Reactors (SFRs) has been formed by OECD/NEA to assess the effect of nuclear data uncertainties on parameters of interest in SFR analysis. In this paper, subexercises of a medium 1000 MWth metallic core (MET-1000) and a large 3600 MWth oxide core (MOX-3600) are tested by a Monte Carlo code MCS to perform uncertainty analysis. Classical perturbation theory and generalized perturbation theory are used to calculate sensitivity coefficients. Uncertainty is calculated by multiplying the sensitivity coefficients and relative covariance matrix from ENDF/B-VII.1 library.
\end{abstract}

KEYWORDS: Uncertainty quantification; MCS; UAM-SFR benchmark;

\section{INTRODUCTION}

Uncertainty analysis in modelling (UAM) for design, operation and safety analysis of Sodium-cooled fast reactors (SFRs) has been formed by OECD/NEA to investigate the effect of nuclear data uncertainties on SFR full core calculations [1]. Since uncertainty analysis of small size model is easy to compare the effect of nuclear data uncertainty, uncertainty analysis for sub-exercises for pin-cell, fuel assembly, and super-cell models is suggested. Recently, uncertainty analysis for sub-exercises has been performed by TSUNAMI of SCALE code system [2] and SARAX/UNICORN [3].

In this paper, the effect of nuclear data uncertainty has been assessed for the sub-exercises in the UAMSFR benchmark. A Monte Carlo code MCS is used as a computational tool for neuron transport calculation and sensitivity/uncertainty (S/U) analysis. MCS [4] is a high-fidelity Monte Carlo particle transport code that has been developed at Ulsan National Institute of Science and Technology (UNIST). It has capabilities of thermal-hydraulics feedback, on-the-fly cross section treatment and depletion calculation. S/U analysis capability using classical perturbation theory (CPT) and generalized perturbation theory (GPT) has been implemented and verified by comparing results between MCS and SERPENT-2 [5]. In Section 2, uncertainty quantification method used in this paper will be introduced briefly. In Section 3, uncertainty analysis results by MCS will be presented for the sub-exercises in UAM-SFR benchmark. To investigate top contributors to uncertainties, sensitivity coefficients and uncertainty of nuclide cross section will be compared between MET-1000 and MOX-3600 models. 


\section{UNCERTAINTY QUANTIFICATION METHOD}

A Monte Carlo code MCS is used for uncertainty quantification of UAM-SFR benchmark. MCS has a capability to compute sensitivity coefficients by using CPT and GPT method. Detailed derivation of GPT equations is given in [6]. Sensitivity coefficient represents a fractional change of a target response due to perturbed input parameters such as cross sections in nuclear data. A target response $Q$ can be defined as a multiplication factor, a reaction rate and a reaction rate ratio. The target response $Q$ in a form of a reaction rate ratio can be expressed as

$$
Q=\frac{\left\langle\mathbf{R}_{\mathbf{1}} S\right\rangle}{\left\langle\mathbf{R}_{2} S\right\rangle}
$$

where $\mathbf{R}_{\mathbf{1}}$ and $\mathbf{R}_{\mathbf{2}}$ denote response functions in numerator and denominator, respectively. $S$ is a fission source density, which is a neutron particle simulated in Monte Carlo calculation. The sensitivity coefficients of a target response $Q, S_{x}^{Q}$, can be expressed as [6]

$$
S_{x}^{Q}=\frac{x}{\Delta x} \frac{\Delta Q}{Q}=\frac{\left\langle\Delta \mathbf{R}_{1} S\right\rangle}{\left\langle\mathbf{R}_{\mathbf{1}} S\right\rangle}-\frac{\left\langle\Delta \mathbf{R}_{2} S\right\rangle}{\left\langle\mathbf{R}_{\mathbf{2}} S\right\rangle}+\frac{\left\langle\mathbf{R}_{\mathbf{1}} \Delta S\right\rangle}{\left\langle\mathbf{R}_{1} S\right\rangle}-\frac{\left\langle\mathbf{R}_{\mathbf{2}} \Delta S\right\rangle}{\left\langle\mathbf{R}_{\mathbf{2}} S\right\rangle},
$$

where input parameter $x$ represents cross sections in nuclear data. On the right-hand side of Eq. (2), the first two terms containing $\Delta \mathrm{R}$ represent perturbed operator effect. The last two terms containing $\Delta S$ represent perturbed source effect. The perturbed operator effect can be computed by differential operator sampling method. It is difficult to compute the perturbed source effect due to presence of $\Delta S$. Choi et al. [6] derived GPT equations including the perturbed source effect with $\Delta S$-free condition. In a Monte Carlo calculation, the sensitivity coefficients can be calculated by [6]

$$
S_{x}^{Q}=\frac{\left\langle\Delta \mathbf{R}_{1} S\right\rangle}{\left\langle\mathbf{R}_{1} S\right\rangle}-\frac{\left\langle\Delta \mathbf{R}_{2} S\right\rangle}{\left\langle\mathbf{R}_{2} S\right\rangle}+\frac{1}{k} \sum_{i=0}^{n}\left[\frac{\left\langle\mathbf{R}_{1}(\mathbf{H} / k)^{i} \Delta \mathbf{H} S\right\rangle}{\left\langle\mathbf{R}_{1} S\right\rangle}-\frac{\left\langle\mathbf{R}_{2}(\mathbf{H} / k)^{i} \Delta \mathbf{H} S\right\rangle}{\left\langle\mathbf{R}_{2} S\right\rangle}\right],
$$

where $i$ is a cycle index representing $i$-th generation from a current generation. $n$ is a convergence interval for adjoint function. $\mathbf{H}$ is a fission operator. Sensitivity coefficients calculated from Eq. (3) are used to uncertainty quantification by using a sandwich rule [7]. The Sandwich rule is the first order uncertainty propagation method which is expressed as

$$
u_{Q}^{2}=S_{x_{1}}^{Q} C_{x_{1}, x_{2}}\left(S_{x_{2}}^{Q}\right)^{T}
$$

where $u_{Q}$ is uncertainty of response $Q . C_{x 1, x 2}$ is a relative covariance matrix of $x_{1}$ and $x_{2}$, which can be obtained from nuclear data library. The energy group structure must be consistent between sensitivity coefficients and covariance matrix. In this work, ENDF-B/VII.1 library is used for the continuous energy neutron cross section and 44-group covariance data.

\section{UNCERTAINTY ANALYSIS OF UAM-SFR SUB-EXERCISES}

\subsection{Pin-cell Models}

Uncertainty analysis has been performed for two-dimensional hexagonal pin-cell models. Fig. 1 shows the specification of MET-1000 and MOX-3600 pin-cell models. In a hexagonal pin-cell, cylindrical fuel rod is 
surrounded by sodium coolant. MOX-3600 fuel pin contains a hole at the center of fuel rod. Fuel compositions are also shown in Fig. 1. The fuel compositions are originated from the fuel at the end of equilibrium cycle (EOEC) [1]. MET-1000 fuel consists of uranium-transuranics (U-TRU) with zirconium and natural molybdenum. MOX-3600 fuel consists of U-TRU oxide fuel with natural molybdenum. The natural molybdenum in both fuel material represents a fission product.

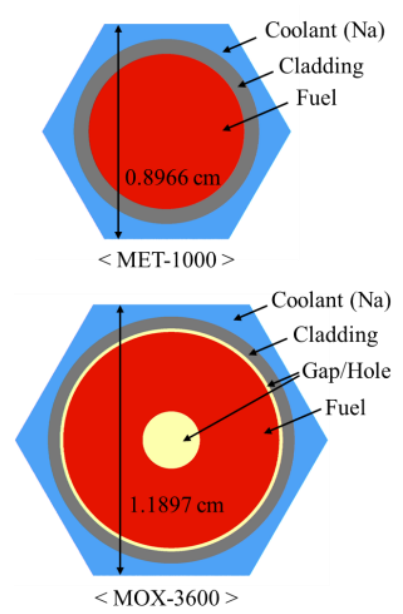

\begin{tabular}{|c|c|c|c|c|c|}
\hline Nuclide & MET-1000 & MOX-3600 & Nuclide & MET-1000 & MOX-3600 \\
\hline O-16 & - & $4.2825 \mathrm{E}-02$ & Am-241 & $8.8828 \mathrm{E}-05$ & $3.6901 \mathrm{E}-05$ \\
\hline $\mathrm{U}-234$ & $1.7210 \mathrm{E}-06$ & $2.1672 \mathrm{E}-06$ & Am-242g & - & $1.7558 \mathrm{E}-08$ \\
\hline $\mathrm{U}-235$ & $2.2106 \mathrm{E}-05$ & $2.1336 \mathrm{E}-05$ & Am-242m & $8.9023 \mathrm{E}-06$ & $1.9169 \mathrm{E}-06$ \\
\hline $\mathrm{U}-236$ & $3.8904 \mathrm{E}-06$ & $6.3334 \mathrm{E}-06$ & Am-243 & $9.1054 \mathrm{E}-05$ & $4.0860 \mathrm{E}-05$ \\
\hline $\mathrm{U}-238$ & $1.8774 \mathrm{E}-02$ & $1.7571 \mathrm{E}-02$ & $\mathrm{Cm}-242$ & $7.4075 \mathrm{E}-06$ & $3.2303 \mathrm{E}-06$ \\
\hline $\mathrm{Np}-237$ & $4.2264 \mathrm{E}-05$ & $7.5991 \mathrm{E}-06$ & $\mathrm{Cm}-243$ & $7.7307 \mathrm{E}-07$ & $2.4680 \mathrm{E}-07$ \\
\hline $\mathrm{Np}-239$ & - & $5.3141 \mathrm{E}-06$ & $\mathrm{Cm}-244$ & $7.1010 \mathrm{E}-05$ & $1.1034 \mathrm{E}-05$ \\
\hline $\mathrm{Pu}-236$ & $7.3569 \mathrm{E}-10$ & - & $\mathrm{Cm}-245$ & $1.5116 \mathrm{E}-05$ & $9.4349 \mathrm{E}-07$ \\
\hline $\mathrm{Pu}-238$ & $1.0560 \mathrm{E}-04$ & $7.4795 \mathrm{E}-05$ & $\mathrm{Cm}-246$ & $9.1659 \mathrm{E}-06$ & $4.7941 \mathrm{E}-08$ \\
\hline $\mathrm{Pu}-239$ & $2.1525 \mathrm{E}-03$ & $1.9750 \mathrm{E}-03$ & $\mathrm{Cm}-247$ & - & $1.6226 \mathrm{E}-09$ \\
\hline $\mathrm{Pu}-240$ & $1.2570 \mathrm{E}-03$ & $1.0276 \mathrm{E}-03$ & $\mathrm{Zr}$ & $7.2802 \mathrm{E}-03$ & - \\
\hline $\mathrm{Pu}-241$ & $1.8043 \mathrm{E}-04$ & $1.8497 \mathrm{E}-04$ & $\mathrm{Mo}$ & $2.7287 \mathrm{E}-03$ & $4.5802 \mathrm{E}-03$ \\
\hline $\mathrm{Pu}-242$ & $2.7677 \mathrm{E}-04$ & $3.0146 \mathrm{E}-04$ & & & \\
\hline
\end{tabular}

Figure 1. Configuration of pin-cell models, and fuel composition in \#/barn-cm

For the pin-cell models, uncertainties are calculated with respect to $k_{\infty}$, one-group microscopic and macroscopic cross sections. The macroscopic cross section is tallied only in a fuel region. Table I shows $k_{\infty}$ and its uncertainty results for pin-cell models. The $k_{\infty}$ uncertainties are $1.44 \%$ and $1.64 \%$ in MET-1000 and MOX-3600, respectively. The $k_{\infty}$ uncertainties are twice compared to the ones of light water reactor cases [8]. Fig. 2 shows the uncertainty summary of the pin-cell models. The uncertainties are in range from $0.5 \%$ to $17.9 \%$. The largest one is the $\sigma_{(n, \gamma), P u 241}$ uncertainty of $17.9 \%$ and $12.5 \%$ in MET-1000 and MOX3600 , respectively.

Table I. Pin-cell multiplication factor results

\begin{tabular}{|c|c|c|}
\hline & $k_{\infty} \pm 1 \sigma$ & Uncertainty $\left(\Delta k_{\infty} / k_{\infty}\right)$ \\
\hline MET-1000 & $1.36016 \pm 0.00012$ & $1.44 \%$ \\
\hline MOX-3600 & $1.19196 \pm 0.00010$ & $1.64 \%$ \\
\hline
\end{tabular}

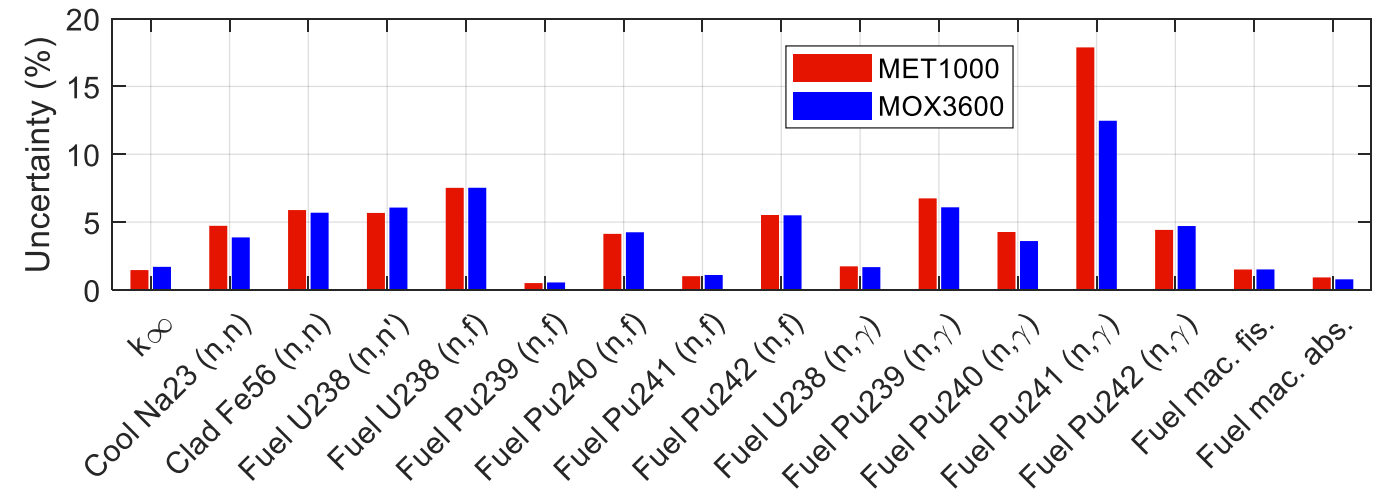

Figure 2. Uncertainty summary of pin-cell models 
Fig. 3 and Fig. 4 show nuclide-reaction pairs that largely contributes to $k_{\infty}$ and $\sigma_{(n, \gamma), P u 241}$ uncertainty. Top three contributors to $k_{\infty}$ uncertainty are shown in Fig. 3a. The uncertainty contribution of $\sigma_{\left(n, n^{\prime}\right), U 238}$ is dominant to $k_{\infty}$ uncertainties in MET-1000 and MOX-3600. Sensitivity coefficient and relative standard deviation (RSD) of $\sigma_{\left(n, n^{\prime}\right), U 238}$ are shown in Fig. 3b. Negative peak of the sensitivity coefficient is shown in fast energy region. Although size of the negative sensitivity peak is less than 0.08 , the large RSD of $20 \%$ makes $\sigma_{\left(n, n^{\prime}\right), U 238}$ as a top contributor to $k_{\infty}$ uncertainty. Fig. 4a shows top contributors to $\sigma_{(n, \gamma), P u 241}$ uncertainty. The largest contribution is caused by $\sigma_{(n, \gamma), P u 241}$. In this case, large sensitivity coefficient and RSD of $\sigma_{(n, \gamma), P u 241}$ makes huge uncertainty contribution as $17.8 \%$ and $12.4 \%$ in MET-1000 and MOX3600 , respectively.

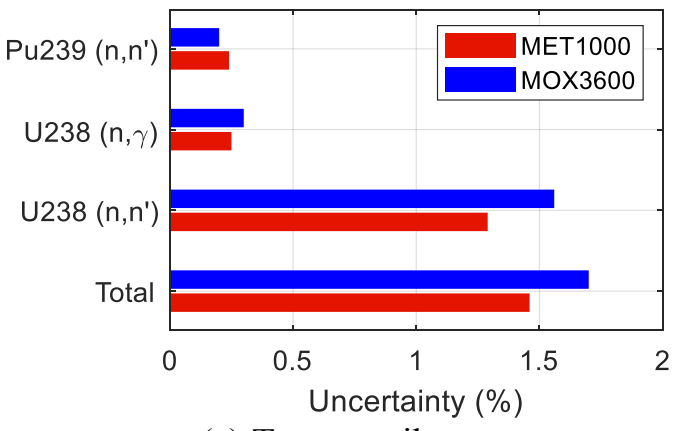

(a) Top contributors

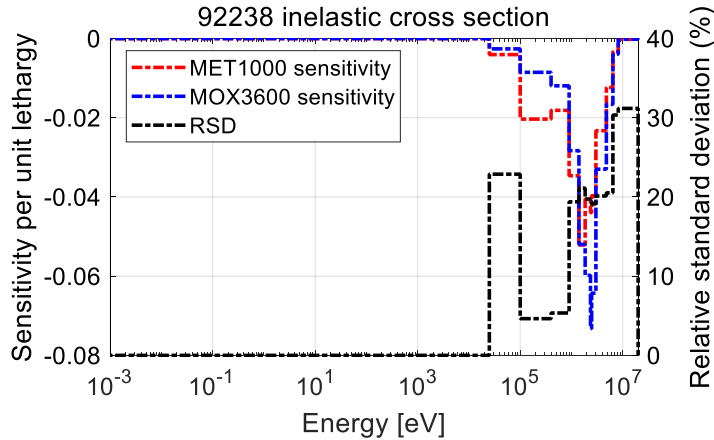

(b) Sensitivity coefficient and RSD

Figure 3. Uncertainty contributions to $\boldsymbol{k}_{\infty}$ uncertainty

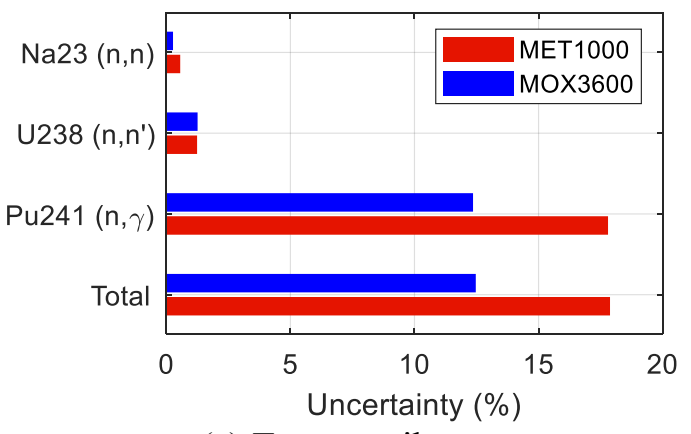

(a) Top contributors

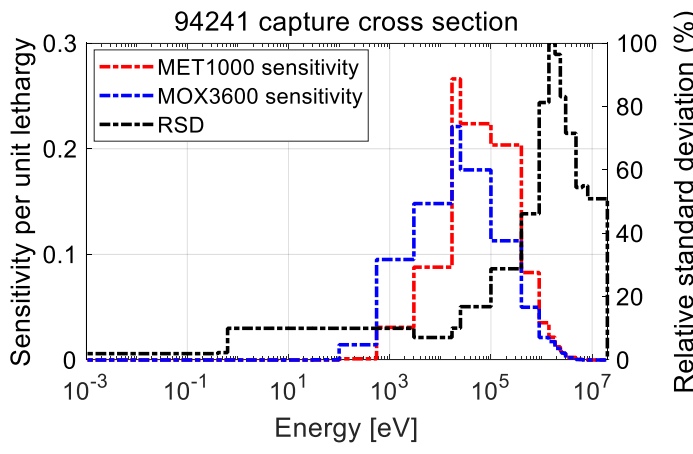

(b) Sensitivity coefficient and RSD

Figure 4. Uncertainty contributions to $\sigma_{(n, \gamma), P u 241}$ uncertainty

\subsection{Fuel Assembly models}

Fig. 5 shows the configuration of assembly models. There are 271 fuel pins in the assembly, and its geometry and material compositions are same with the ones used in pin-cell models. Fig. 6 shows neutron spectrum of fuel assembly models. It is shown that the neutron spectrum of MET-1000 is harder than the one of MOX-3600. For the fuel assembly models, uncertainties of $k_{\infty}$ and homogenized macroscopic 4group cross sections are calculated. The upper energy boundaries for the 4-group structure are $20 \mathrm{MeV}, 820$ $\mathrm{keV}, 110 \mathrm{keV}$ and $15 \mathrm{keV}$. 


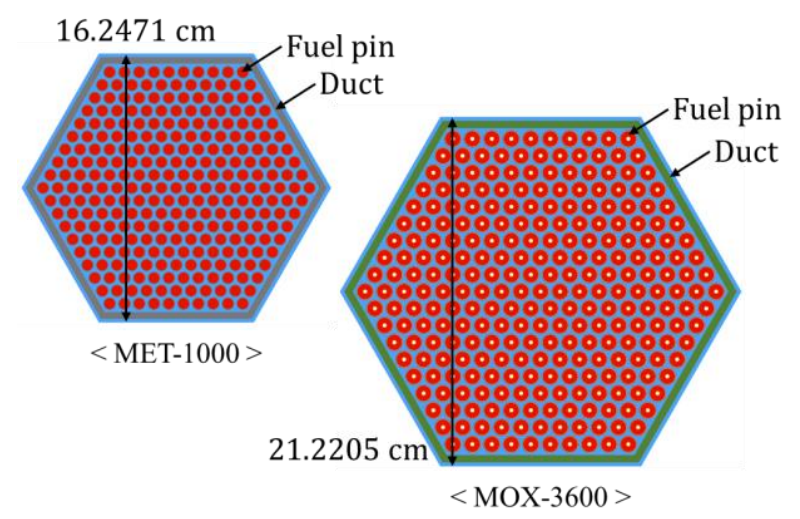

Figure 5. Configuration of fuel assembly models

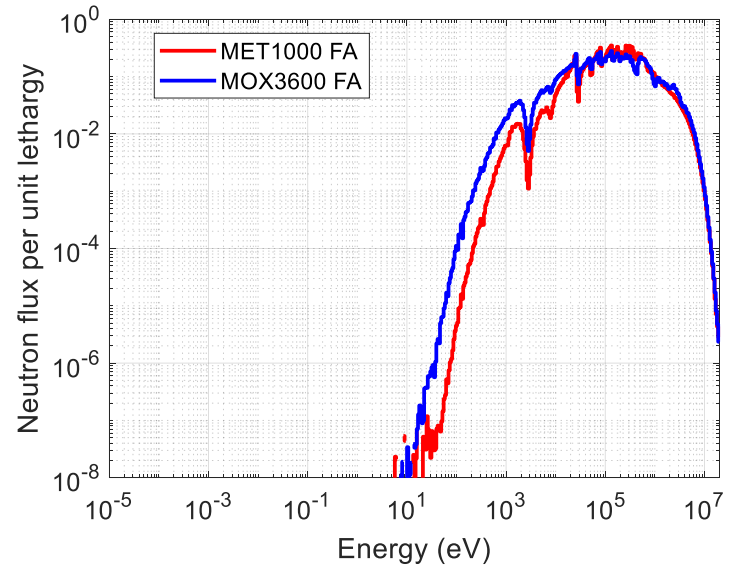

Figure 6. Neutron spectrum of fuel assembly

Table II. Fuel assembly multiplication factor results

\begin{tabular}{|c|c|c|}
\hline & $k_{\infty} \pm \sigma$ & Uncertainty $\left(\Delta k_{\infty} / k_{\infty}\right)$ \\
\hline MET-1000 & $1.28143 \pm 0.00011$ & $1.34 \%$ \\
\hline MOX-3600 & $1.14714 \pm 0.00010$ & $1.44 \%$ \\
\hline
\end{tabular}

Table II shows $k_{\infty}$ and its uncertainty results. The $k_{\infty}$ uncertainties are on a similar level with the ones of pin-cell models. Top contributor to $k_{\infty}$ uncertainties is also $\sigma_{\left(n, n^{\prime}\right), U 238}$ having similar sensitivity profile. Fig. 7 shows the uncertainty summary of fuel assembly models. The uncertainties are in range from $0.5 \%$ to $2.6 \%$.

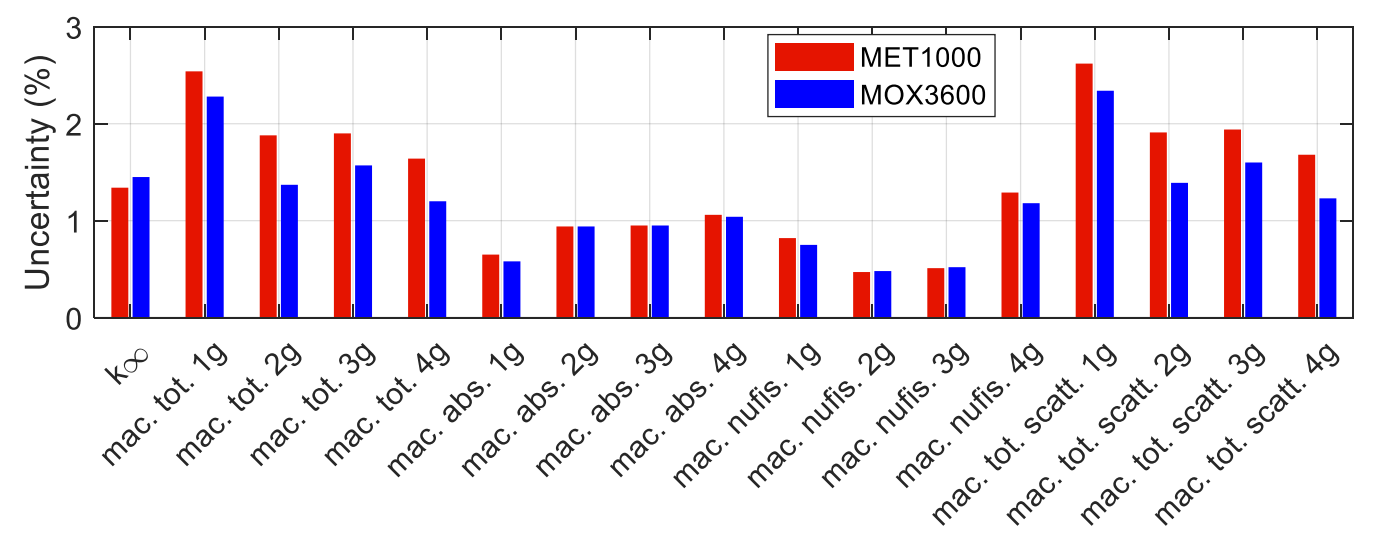

Figure 7. Uncertainty summary of fuel assembly models

In Fig. 8a, top contributors to $\Sigma_{s c a t t, 2 g}$ uncertainty are shown. There is an additional contribution from O16 only in MOX-3600 fuel. The contribution of $\sigma_{(n, n), F e 56}$ is dominant to $\Sigma_{s c a t t, 2 g}$ uncertainty. The difference of uncertainty contribution is caused by different sensitivity coefficient shown in Fig. $8 \mathrm{~b}$. The different sensitivity profile can be explained by harder neutron spectrum of MET-1000. 


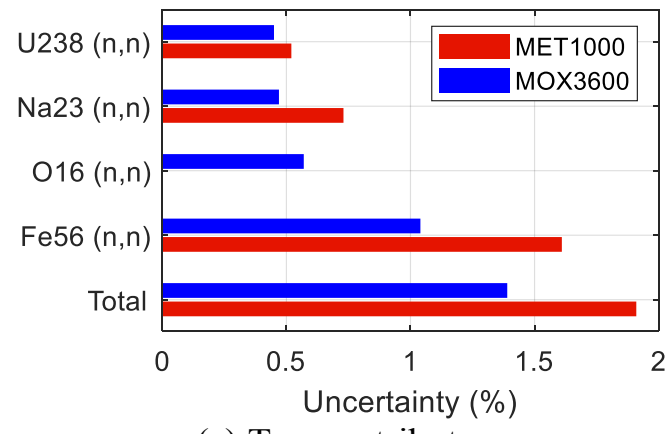

(a) Top contributors

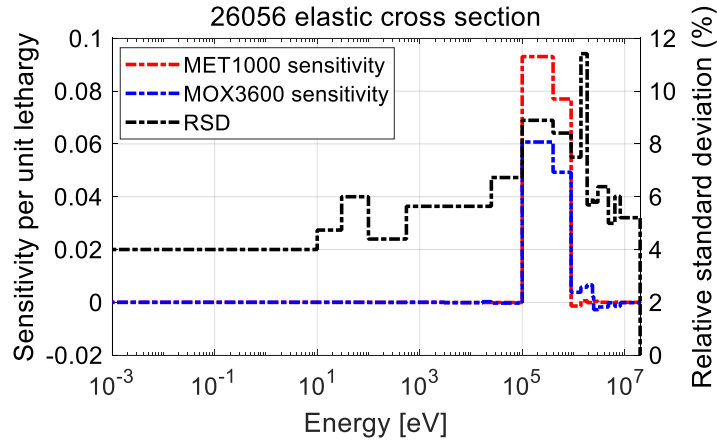

(b) Sensitivity coefficient and RSD

Figure 8. Uncertainty contributions to $\Sigma_{s c a t t, 2 g}$ uncertainty

\subsection{Super-cell models}

The configuration of super-cell models is shown in Fig. 9. A primary control assembly is located at the center of super-cell, and it is surrounded by fuel assemblies to represent fuel-to-absorber ratio of full core. The boron carbide $\left(\mathrm{B}_{4} \mathrm{C}\right)$ is used in absorber rod, and its material composition is shown in Table III. Table IV shows $k_{\infty}$ and its uncertainty results. As it is observed in fuel assembly models, $k_{\infty}$ uncertainties are similar with the ones of pin-cell models. The top contributor to $k_{\infty}$ uncertainty is same as $\sigma_{\left(n, n^{\prime}\right), U 238}$.

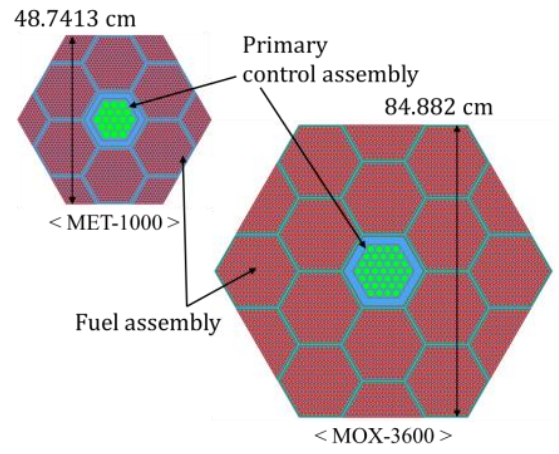

Figure 9. Configuration of supercell models
Table III. Absorber rod material composition in \#/barn-cm

\begin{tabular}{|c|c|c|}
\hline Nuclide & MET-1000 & MOX-3600 \\
\hline C & $1.9657 \mathrm{E}-02$ & $2.70 \mathrm{E}-02$ \\
\hline B-10 & $1.5018 \mathrm{E}-02$ & $2.32 \mathrm{E}-02$ \\
\hline B-11 & $6.3609 \mathrm{E}-02$ & $8.49 \mathrm{E}-02$ \\
\hline
\end{tabular}

Table IV. Super-cell multiplication factor results

\begin{tabular}{|c|c|c|}
\hline & $k_{\infty} \pm \sigma$ & Uncertainty $\left(\Delta k_{\infty} / k_{\infty}\right)$ \\
\hline MET-1000 & $1.08484 \pm 0.00015$ & $1.45 \%$ \\
\hline MOX-3600 & $1.07728 \pm 0.00012$ & $1.47 \%$ \\
\hline
\end{tabular}

The uncertainty summary for super-cell models is shown in Fig. 10. The macroscopic cross sections are homogenized in the primary control assembly. The same energy group structure used in fuel assembly models are used for the homogenized 4-group macroscopic cross sections. Fig. 10 shows the uncertainty summary of super-cell models. Uncertainties are in range from $0.2 \%$ to $1.9 \%$. 


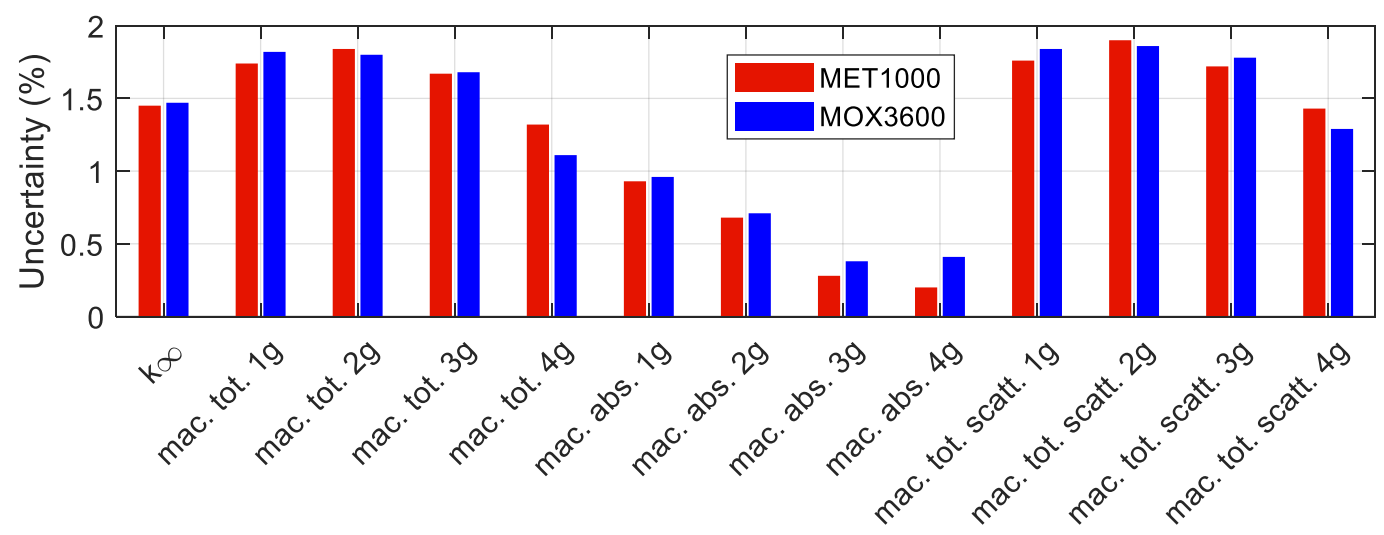

Figure 10. Uncertainty summary of super-cell models

Fig. 11 and Fig. 12 shows top contributors to $\Sigma_{a b s, 1 g}$ and $\Sigma_{a b s, 2 g}$ uncertainties, respectively. The contribution of B-10 in absorber rod is dominant for both uncertainties. However, the way it contributes to the uncertainty is different. While the large RSD of $\sigma_{(n, p), B 10}$ makes large contribution to $\Sigma_{a b s, 1 g}$ uncertainty, large sensitivity coefficient of $\sigma_{(n, \alpha), B 10}$ makes large contribution to $\Sigma_{a b s, 2 g}$ uncertainty. In Fig. 12a, negative contribution to $\Sigma_{a b s, 2 g}$ uncertainty is observed due to negative correlation between $\sigma_{(n, n), B 10}$ and $\sigma_{(n, \gamma), B 10}$.

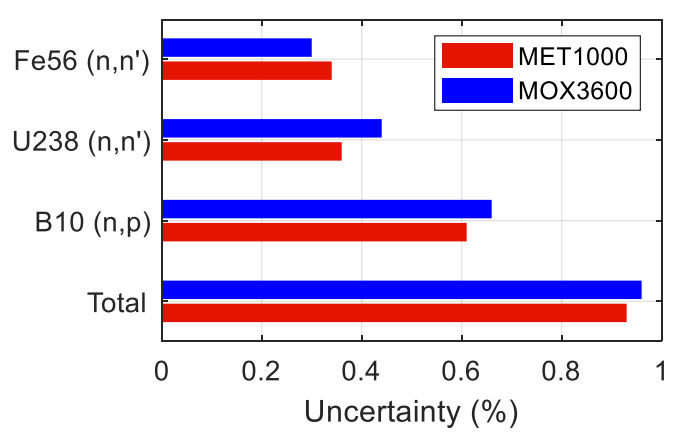

(a) Top contributors

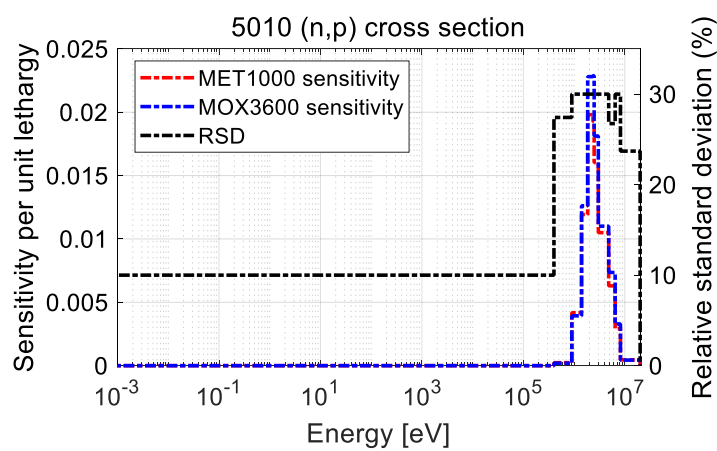

(b) Sensitivity coefficient and RSD

Figure 11. Uncertainty contributions to $\Sigma_{a b s, 1 g}$ uncertainty

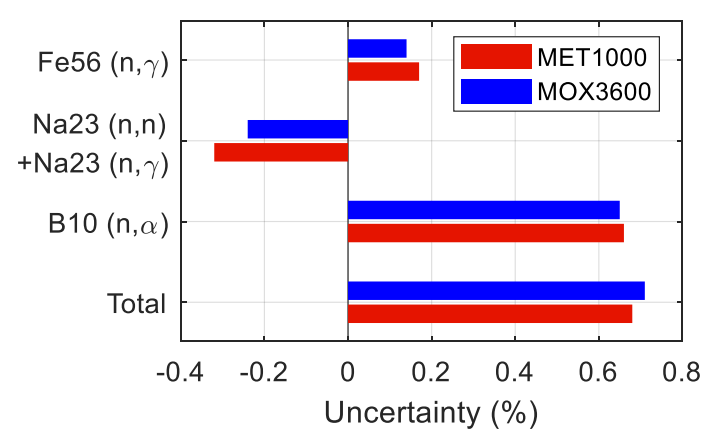

(a) Top contributors

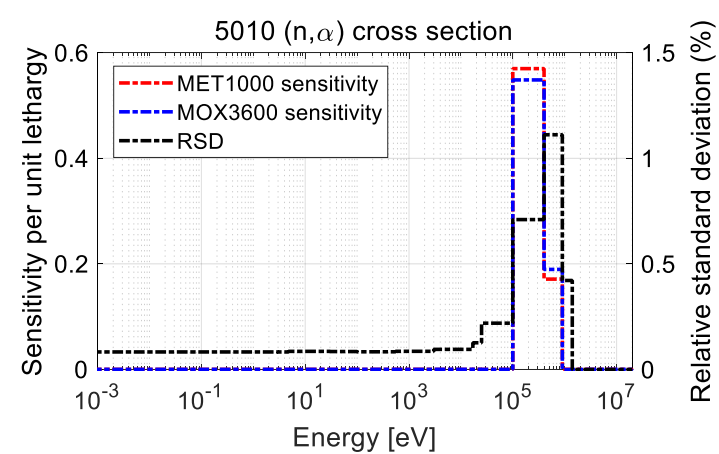

(b) Sensitivity coefficient and RSD

Figure 12. Uncertainty contributions to $\Sigma_{a b s, 2 g}$ uncertainty 


\section{CONCLUSIONS}

In this work, uncertainty analysis has been performed to quantify the effect of nuclear data uncertainty on $k_{\infty}$, microscopic and macroscopic cross sections in UAM-SFR benchmark. A Monte Carlo code MCS has been used as an uncertainty quantification tool. The $k_{\infty}$ uncertainty is about $1.5 \%$ in pin-cell, fuel assembly and super-cell models of MET-1000 and MOX-3600. It is twice of the $k_{\infty}$ uncertainty in light water reactor cases. Top contributor to the $k_{\infty}$ uncertainty is $\sigma_{\left(n, n^{\prime}\right), \mathrm{U} 238}$. The contribution is caused by the large RSD of $\sigma_{\left(n, n^{\prime}\right), \mathrm{U} 238}$ in fast energy region. It is possible to reduce the $k_{\infty}$ uncertainty effectively by decreasing uncertainties in $\sigma_{\left(n, n^{\prime}\right), \mathrm{U} 238}$. The largest uncertainty is $\sigma_{(n, \gamma), \mathrm{Pu} 241}$ uncertainty of $17.9 \%$ and $12.5 \%$ in MET1000 and MOX-3600 pin-cell models, respectively. The main contribution is caused by the large RSD of $\sigma_{(n, \gamma), \mathrm{Pu} 241}$. In fuel assembly models, neutron spectrum of MET-1000 and MOX-3600 are compared. It is shown that the one of MET-1000 is harder than the one of MOX-3600. The harder neutron spectrum causes larger sensitivity coefficient in fast energy region. As a result, the larger uncertainty is calculated by multiplying the larger sensitivity and RSD of cross section in a fast energy region. The uncertainties of 4group macroscopic cross sections are in range from $0.5 \%$ to $2.6 \%$. In a super-cell model, a primary control assembly containing boron carbide absorber rods is located at the center of it. The largest contribution to the uncertainty of $\Sigma_{a b s, 1 g}$ and $\Sigma_{a b s, 2 g}$ is caused by B-10 in absorber rods. The $\sigma_{(n, p), B 10}$ and $\sigma_{(n, \alpha), B 10}$ are top contributors to the uncertainty of $\Sigma_{a b s, 1 g}$ and $\Sigma_{a b s, 2 g}$, respectively. The uncertainties of 4-group homogenized macroscopic cross sections are in range from $0.2 \%$ to $1.9 \%$.

\section{ACKNOWLEDGMENTS}

This work was supported by the National Research Foundation of Korea (NRF) grant funded by the Korea government (MSIT). (No. NRF-2019M2D2A1A03058371).

\section{REFERENCES}

1. AEN-WPRS, "Benchmark for Uncertainty Analysis in Modelling for Design, Operation and Safety Analysis of SFRs," Version 1.6 (2019).

2. F. Bostelmann, B. T. Rearden, W. Zwermann and A. Pautz, "Preliminary SCALE/TSUNAMI Results for the Sub-exercises of the OECD/NEA Benchmark for Uncertainty Analysis in Modeling of SodiumCooled Fast Reactors," Transactions of American Nuclear Society, Orlando, FL, November 11-15, (2018).

3. L. Qiao, Y. Zheng and C. Wan, "Uncertainty Quantification of Sodium-cooled Fast Reactor Based on the UAM-SFR Benchmarks: From Pin-cell to Full Core," Ann. Nucl. Energy, 128, pp. 433-442 (2019).

4. H. Lee, W. Kim, P. Zhang, M. Lemaire, A. Khassenov, Y. Jo, J. Park, J. Yu and D. Lee, "Development of Monte Carlo Multi-physics Code MCS for Power Reactor Analysis," M\&C 2019, Portland, Oregon, August 25-29, pp. 1770-1779 (2019).

5. Y. Jo, C. Kong, A. Cherezov and D. Lee, "Sensitivity and Uncertainty Analysis Capability in MCS for UAM Benchmark," M\&C 2019, Portland, Oregon, August 25-29, pp. 622-630 (2019).

6. S. Choi, H. Shim, C. Kim, "Development of Generalized Perturbation Theory Algorithms for Monte Carlo Eigenvalue Calculations," Nucl. Sci. Eng., 189, pp. 171-187 (2018).

7. M. Pusa, "Perturbation-Theory-Based Sensitivity and Uncertainty Analysis with CASMO-4," Sci. Technol. Nucl. Ins., 2012 (2012).

8. Y. Jo, W. Lee, C. Kong and D. Lee, "Uncertainty Analysis of UAM-LWR Benchmark with MCS," Transactions of the American Nuclear Society, Vol. 121, Washington, D.C., November 17-21 (2019). 